\title{
EDITORIAL
}

\section{You are what you eat or you eat what you are?}

\author{
Nadia Micali ${ }^{1,2,3} \cdot$ Mohamed Abdulkadir $^{1,2}$ \\ Published online: 24 February 2020 \\ (c) Springer-Verlag GmbH Germany, part of Springer Nature 2020
}

This month's issue sees the publication of an interesting study on Attention-Deficit/Hyperactivity Disorder (ADHD) and the gut microbiome. The literature on microbiome and its role in the pathophysiology and manifestation of neuropsychiatric disorders has been proliferating in recent years [1]. Research into the gut microbiome has given us clearer insights into the strong link between the gut and the brain, and the bidirectional relationships between the microbiome and neuropsychiatric disorders.

Of course, the gut microbiome is hugely influenced by nutrition and eating behaviours [2]. The association between eating behaviours and ADHD is well documented. Longitudinal and cross-sectional studies in the general population and in clinical samples show an association between ADHD and/or its symptoms, and over-eating, binge eating (i.e. overeating with loss of control over what is eaten), and eating disorders (bulimia nervosa or binge eating disorder) [3-5]. ADHD symptoms in late childhood were prospectively associated with binge eating at age 14 and 16 years, with late childhood overeating and early adolescent desire for food as mediators [5]. Moreover, evidence suggests that the association between ADHD and related symptoms and eating behaviours is present from early childhood. For example, in a population-based study, ADHD and related symptoms were associated with high levels of food responsiveness, overeating, and food fussiness at age 4 years [6].

Much research has also tried to understand the relationship between ADHD and obesity or overweight (for a review see [7]). It is still debated what the causal mechanisms for these associations are, amongst the possible ones: common

Nadia Micali

nadia.micali@unige.ch

1 Department of Psychiatry, Faculty of Medicine, University of Geneva, Geneva, Switzerland

2 Depatment of Pediatrics, Gynecology and Obstetrics, University of Geneva, Geneva, Switzerland

3 UCL Great Ormond Street Institute of Child Health, University College London, London, UK neurobiological characteristics, such as executive dysfunction (e.g., high impulsivity), shared risk factors (e.g., a genetic or environmental precursor shared by both disorders), or a direct 'causal' link.

New genetic studies suggest that the association between weight, eating disorders and ADHD symptoms might be due to shared genetic liability. Both the ADHD and Body Mass Index (BMI) polygenic risk scores were associated, and both predicted impulsivity and BMI via a cluster of (structural and functional) neuroimaging biomarkers, suggesting evidence of a shared neural substrate for the two associated phenotypes (BMI and ADHD) [8].

Shared genetic factors might explain both ADHD and specific characteristics of the gut microbiota. Indeed the composition of the gut microbiome is affected by multiple factors including the host genetics. This is supported by studies that have shown that the gut microbiome is heritable, and that the abundance of many microbial taxa is influenced by host genetics [9]. For example, in a recently published study, variation in the copy number of the human salivary amylase gene influenced the gut and oral microbiota [9]. Having differential copy numbers of the human salivary amylase gene influences the expression of the amylase enzyme, and therefore the capacity to degrade starch, which in turn impacts the availability of complex carbohydrates in the gut. The difference in availability of complex carbohydrates drives changes in the composition of the intestinal microbiota.

Some authors however, argue that the environment has a substantial greater role than host genetics in shaping up the gut microbiota. A study led by Rothschild et al. [10] found significant similarity among the (gut) microbiomes of genetically unrelated individuals sharing the same household but no significant similarities in related individuals that do not share a history of the same household. What we can learn from these studies is that host genetics do play a role in in shaping up the gut microbiota, however host genetics might only be one factor that shapes individual gut microbiota, with environment also playing a role. 
One alternative hypothesis for the association between altered gut microbiota and ADHD is that genes affect both risk for ADHD, and eating behaviours (such as overeating and binge eating), with altered gut microbiota being just a by-product. Poor nutrition (i.e. a diet low in fiber-a complex carbohydrate, usually found in fruits and vegetableshigh in fats and carbohydrates) is a correlate of eating behaviours such as emotional overeating, food fussiness, binge eating, and altered microbiome might just be a result of poor nutrition. Therefore, gut microbiome might not necessarily lie on the causal pathway to ADHD, but be a correlate of ADHD. Specific gut microbiome characteristics might also in turn worsen ADHD or neuropsychiatric symptoms closing this vicious cycle.

Given these unknowns, describing the gut microbiota identified in this study as differing between children with ADHD and controls as 'biomarkers of ADHD' [https://doi. org/10.1007/s00787-019-01352-2] seems wildly premature. In essence, the complex relationship between nutrition, eating behaviours, weight, and ADHD has not been fully unravelled yet. Disentangling whether it is nutrition and/or eating behaviours that temporally precede ADHD or vice versa is difficult. Adding gut microbiome into this complex mix requires care and cautiousness. Inferring causality in this chain of associations is even harder, and needs to be tested with adequate study designs. The road to unravelling these complex relationships is long and arduous; but big data and very carefully designed studies are needed. This is true of many clinical studies that can provide useful hypotheses but often cannot answer causality questions.

\section{References}

1. Bastiaanssen TFS, Cowan CSM, Claesson MJ, Dinan TG, Cryan JF (2019) Making sense of ... the microbiome in psychiatry. Int J Neuropsychopharmacol 22(1):37-52

2. Kau AL, Ahern PP, Griffin NW, Goodman AL, Gordon JI (2011) Human nutrition, the gut microbiome and the immune system. Nature 474(7351):327-336

3. Geuijen PM, Buitelaar JK, Fliers EA et al (2019) Overweight in family members of probands with ADHD. Eur Child Adolesc Psychiatry 28(12):1659-1669

4. Evans BC, Felton JW, Lagacey MA, Manasse SM, Lejuez CW, Juarascio AS (2019) Impulsivity and affect reactivity prospectively predict disordered eating attitudes in adolescents: a 6-year longitudinal study. Eur Child Adolesc Psychiatry 28(9):1193-1202

5. Sonneville KR, Calzo JP, Horton NJ et al (2015) Childhood hyperactivity/inattention and eating disturbances predict binge eating in adolescence. Psychol Med 45(12):2511-2520

6. Leventakou V, Micali N, Georgiou V et al (2016) Is there an association between eating behaviour and attention-deficit/hyperactivity disorder symptoms in preschool children? J Child Psychol Psychiatry 57(6):676-684

7. Cortese S, Tessari L (2017) Attention-deficit/hyperactivity disorder (ADHD) and obesity: update 2016. Curr Psychiatry Rep 19(1):4-4

8. Barker ED, Ing A, Biondo F et al (2019) Do ADHD-impulsivity and BMI have shared polygenic and neural correlates? Mol Psychiatry. https://doi.org/10.1038/s41380-019-0444-y

9. Poole AC, Goodrich JK, Youngblut ND et al (2019) Human salivary amylase gene copy number impacts oral and gut microbiomes. Cell Host Microbe 25(4):553-564 (e557)

10. Rothschild D, Weissbrod O, Barkan E et al (2018) Environment dominates over host genetics in shaping human gut microbiota. Nature 555(7695):210-215 